\title{
Decision Making in Multi-Issue e-Market Auction Using Fuzzy Techniques and Negotiable Attitudes
}

\section{Madhu Goyal ${ }^{1}$, Jie $\mathrm{Lu}^{2}$ and Guang Zhang ${ }^{3}$}

\author{
University of Technology, Sydney \\ PO BOX 123 Broadway NSW 2007 Australia \\ ${ }^{1}$ madhu@it.uts.edu.au, ${ }^{2}$ jielu@it.uts.edu.au, , ${ }^{3}$ zhangg@it.uts.edu.au
}

Received 16 April 2007; received in revised form 17 January 2008; accepted 14 April 2008

\begin{abstract}
Online auctions are one of the most effective ways of negotiation of salable goods over the internet. Software agents are increasingly being used to represent humans in online auctions. These agents can systematically monitor a wide variety of auctions and can make rapid decisions about what bids to place in what auctions. To be successful in open multi-agent environments, agents must be capable of adapting different strategies and tactics to their prevailing circumstances. This paper presents a software test-bed for studying autonomous bidding strategies in simulated auctions for procuring goods. It shows that agents' bidding strategy explore the attitudes and behaviors that help agents to manage dynamic assessment of prices of goods given the different criteria and scenario conditions. Our agent also uses fuzzy techniques for the decision making: to make decisions about the outcome of auctions, and to alter the agent's bidding strategy in response to the different criteria and market conditions.
\end{abstract}

Key words: Agents, Attitudes, Bidding strategy, Fuzzy sets, Linguistic variables 


\section{Introduction}

Online auctions (e.g. eBay, Amazon etc.) are one of the most effective ways of negotiation of sellable goods over the internet. In these on-line auctions buyers are faced with difficult task of deciding amount to bid in order to get the desired item matching their preferences. Due to the nature of the tasks involved in e-commerce, intelligent agent technology for the past decade has so far played an important role in its development of software agents in ecommerce transactions. Intelligent agents are systems that inhabit a dynamic, unpredictable environment in which they try to satisfy a set of time dependent goals or motivations. As compared to Expert systems [27], which are typically disembodied 'expertise' about some (abstract) domain of discourse agents are situated in an environment. Negotiation using agents will become a key part of next generation electronic commerce. The agents by playing the role of buyer, seller, mediator and facilitator automate different business negotiations. In automated negotiations, the agents prepare bids and evaluate offers in order to obtain the maximum return for the parties they represent. The agents can use different auction mechanisms (e.g. English, Dutch, Vickery) for procurement of goods or reaching agreement between agents. The agent makes decisions on behalf of buyer and endeavours to guarantee the delivery of item according to the buyer's preferences. Such automated negotiations lead to dynamic pricing which ensures that goods and services are allocated to the entity that values them most highly. To this end, a number of researchers [1], [2], [4], [6], [13] have reported different frameworks that help an autonomous agent to tackle the problem of bidding in auctions.

Current auction mechanisms are simple price finding (first-generation) mechanisms. Considering that product differentiation has become a widespread strategy to defy comparison-shopping on electronic markets, there will be a need for more complex negotiation protocols. In particular, for more complex goods and services, multi-attribute negotiations will become an important feature of electronic commerce [6], [13]. Therefore, many experts stress the importance of developing multi-attribute negotiation protocols in electronic commerce. In multi-attribute auctions in contrast to conventional single-attribute auctions, it is not obvious for the bidder right from the start which combination of attributes provides the highest overall utility for the bid taker. Nevertheless, appropriate software tool or agent for the bidder plays a crucial role when there are three or more negotiable attributes. However, it also requires the skills of a good researcher to develop such a successful software agent, which has an appropriate negotiation methodology for a bidder in electronic marketplace.

Currently, no single implementation satisfies all the criteria, but there are several promising results for bargaining "intelligent" agents. Drawing on theoretical economics [4] and game theory [17], [20], much academic work has been carried out on negotiation strategies, though as yet only simple strategies have been deployed. Strategies exist for many negotiating price in many-to-many environments and for participating in multiple one-to-many auctions simultaneously. Preliminary simulation-based studies have been carried out to determine the effectiveness of such strategies, but the extensive testing that would be required by the industry has yet to be undertaken. However, given the potential and the importance of using agents in online auction setting, there has been considerable research endeavour in developing bidding strategies for different types of agents in different types of auctions. This paper reports on the work in developing a bidding agent that has range of strategies that it can employ depending on the user's aim and the criteria. In this paper, a method of bidding is designed in a multi-agent dynamic world based on the concept of attitude. Attitude is a mental construct similar to that of commitment and intention. However, the notion of commitments as used in Al is too weak for agents solving problems in dynamic worlds. We propose that when agents are solving problems they must be guided by appropriate attitudes towards world objects and their activities. Thus, in our formulation, agents will adopt a definite attitude towards the bidding activity while performing that activity, even if the activity may not succeed in a dynamic world. The adopted attitude will guide the agent in responding to various different situations. For this reason, agents inhabiting in such complex multi-agent dynamic worlds, while solving problems must be holding appropriate attitudes towards their physical and mental activities. The advantage of using attitudes is that the agents will be modeled and developed as bidders in real life auction environments. It will be noticed that attitude based agents outperform the agents without attitude in highly dynamic bidding environments. Agents, which adopt attitudes, behave more flexibly and efficiently than agents without attitude and adapt more easily to dynamic situations. Another unique idea presented in this project will be to deal quantitatively the imprecision or uncertainty of attitudes, the fuzzy theory will be used. The fuzzy theory will provide attitude based software agents resources in the decision making process of bidding agents. The developed software agent will also use fuzzy techniques for the decision making: to make decisions about the outcome of auctions, and to alter the agent's bidding strategy in highly dynamic market conditions. The fuzzy theory will also help to quantify attitudes and to deal with imprecision or uncertainty. In our model, the attitudes will be represented by linguistic terms [31]. The concept of linguistic variables is useful in dealing with situations that are too complex or too illdefined to be reasonably described in conventional quantitative expressions. Since these linguistic terms reflect the uncertainty, inaccuracy and fuzziness of decision makers or bidders, fuzzy number and fuzzy operations can be directly applied to deal with them. We choose to adopt a fuzzy logic based approach, in particular, because we aim to develop a bidding agent that can cope with the uncertainties in a timely manner. The fuzzy techniques have proven to be successful in a wide range of domains with these characteristics e.g. fuzzy control to drive car-like vehicles [10], medical diagnosis [29], vehicle dispatching [26], and electrical power distribution [21]. This paper thus proposes two unique ideas i.e. first a novel attitude based agents are used in a bidding environment; second fuzzy theory is used for bidding in highly dynamic bidding environment. 


\section{Related Work}

There have also been several approaches from computer scientists for developing intelligent software methods and protocols for automated negotiation. In particular, the first trading agent competition (TAC) was held in Boston in July 2000 [14]. TAC agents acted as simulated travel agents and had to procure goods for their clients in different types of auctions, bidding against autonomous agents. Priest et al. [22] proposed an algorithm design for agents that participate in multiple simultaneous English auctions. The algorithm proposes a coordination mechanism to be used in an environment where all the auctions terminate simultaneously, and a learning method to tackle auctions that terminate at different times. Byde et al. [6] presented another decision theoretic framework that an autonomous agent can use to bid effectively across multiple auctions with various protocols. The framework uses an approximation function that provides an estimate of the expected utility of participating in the set of future auctions and it can be employed to purchase single or multiple items. Anthony et al. [1] also propose an approach for agents to bid for a single item in English, Dutch, and Vickrey auctions. The agent decides what to bid based on four parameters: i) the remaining time; ii) the number of remaining auctions; iii) the desire for bargain; and iv) the desperateness of the agent. The overall strategy is to combine these four tactics using a set of relative weights provided by the user. In an extension to this model [2], a genetic algorithm is used to search the effective strategies so that an agent can behave appropriately according to its assessment of its prevailing circumstances. The machine learning techniques [24] are also used to obtain a model of the price dynamics based on the past data (e.g., the data in the seeding round) to predict the closing prices of the hotels in the future. It also uses mixed-integer linear programming to find the optimal allocation of the goods.

We now discuss how the fuzzy techniques are used to manage an agent's interactions. Faratin et al. [11] used fuzzy similarity to compute tradeoffs among multiple attributes during bilateral negotiations. In this paper, fuzzy techniques are used to deal with a bilateral negotiation and the algorithm aims to find a win-win solution for both parties. Kowalcyzk and Bui [15], [16] modeled the multi-issue negotiation process as a fuzzy constraint satisfaction problem. Their approach performs negotiation on individual solutions one at a time. During negotiation, an agent evaluates the offers, relaxes the preferences, constraints and makes counter-offers to find an agreement for both parties. The issues negotiated over actually correspond to the constrained variables and the preferences, constraints and each party's objectives are expressed as fuzzy constraints over these issues. Using this method, the FCSP is to find a solution that maximizes the satisfaction of all constraints of the parties. Luo et al. [18], [19] developed a fuzzy constraint based framework for bilateral multi-issue negotiations in semi-competitive trading environments. The framework is expressed via two knowledge models, one for the seller agent and one for the buyer agent. The seller agent's domain knowledge consists of its multi-dimensional representation of products or services it offers. The buyer agent's domain knowledge consists of the buyer's requirement/preference model (a prioritized fuzzy constraint problem) and buyer's profile model (fuzzy truth propositions). The buyer and seller agents exchange offers and counter-offers with additional constraints revealed or existing constraints being relaxed. Finally, a solution is found if there is one.

Different researchers have also provided alternatives to fuzzy reasoning for coping with the uncertainties in bidding. For example, the possibility-based approach [20] has been used to perform multi-agent reasoning under uncertainty for bilateral negotiations. In this paper, uncertainties due to the lack of knowledge about other agents' behaviors are modeled by possibility distributions. The Bayesian learning method [30] has also been used to model multi-issue negotiation in a sequential decision making model. In this paper, a Bayesian network is used to update the knowledge and belief each agent has about the environment and other agents, and offers and counter-offers between agents during bilateral negotiations are generated based on Bayesian probabilities.

\section{Attitude Based Agent}

In recent years, many researchers and practitioners have focused on the design of market architectures [14], [24] for electronic commerce, and on protocols governing the interaction of self-interested agents engaged in such transactions. While providing support for direct agent negotiation, the existing architectures for multi-agent virtual markets usually lack explicit decision making tools for handling multiple and varied negotiation. We have proposed an attitude based agent architecture (Figure 1) and discuss its features, which guide the agents in automated bidding The agent architecture consists of the following modules:

Sensor: The function of the sensor is to sense the changes in the world (auction) and accordingly update the bids pre-processor. The sensor aims to determine the state of auction both before an auction starts and during the course of the auction.

Bids Pre-processor: The function of bids pre-processor is to get the prices of different goods, number of goods it may possibly own and its current active bids.

World Model: The bidding agent maintains the world model which contains its perceptions about the state of world.

Attitude Generator: An agent responds to events occurring in the world by generating attitudes through the attitude generator. When the sensor senses the changes in the world, the agent updates its world model and forms the 
various beliefs. The attitude generator is a module, which generates attitudes to specify the right type of behaviour required for bidding activity.

Bid Generator and Bid Pool: The function of the goal generator is to generate different bids under different bidding criteria's. The bids generated are stored in bid pool for further selection.

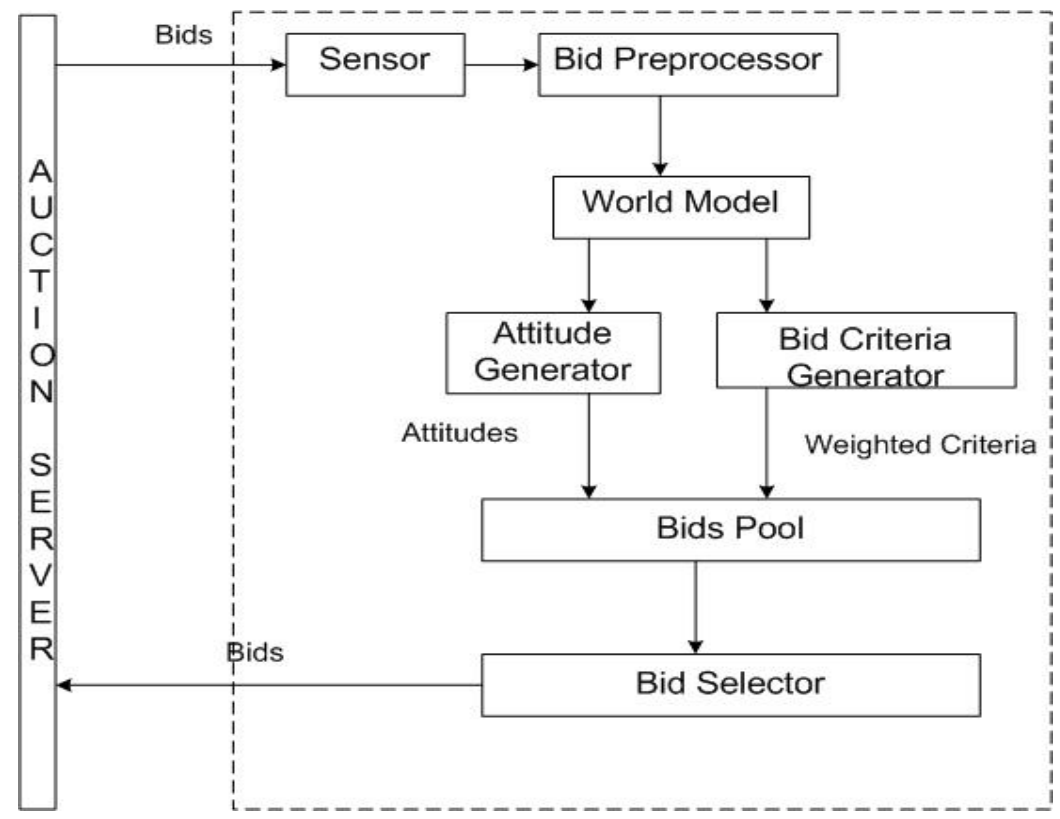

Figure 1: Attitude Based Agent

Bid Selector: The function of the bid selector is to select the best possible bid from the number of bids in bids pool. The agent uses attitude based bidding strategy (section 3 ) to select the best possible bid.

\subsection{Definition of Attitude}

Attitude is a learned predisposition to respond in a consistently favourable or unfavourable manner with respect to a given object [9]. In other words, the attitude is a preparation in advance of the actual response, it constitutes an important determinant of the ensuing behaviour. However this definition seems too abstract for computational purposes. In Al, the fundamental notions to generate the desirable behaviors of the agents often include goals, beliefs, intentions, and commitments. Goal is a subset of states, and belief is a proposition that is held as true by an agent. Bratman [3] addresses the problem of defining the nature of intentions. Crucial to his argument is the subtle distinction between doing something intentionally and intending to do something. The former case might be phrased as deliberately doing an action, while intending to do something means one may not be performing the action in order to achieve it. Cohen and Levesque [7], on the other hand, developed a logic in which intention is defined. They define the notion of individual commitment as a persistent goal, and an intention is defined to be a commitment to act in a certain mental state of believing throughout what he is doing. Thus to provide a definition of attitude that is concrete enough for computational purposes, we model attitude using goals, beliefs, intentions and commitments. From the Fishbein's [9] definition it is clear that when an attitude is adopted, an agent has to exhibit an appropriate behaviour (predisposition means behave in a particular way). The exhibited behaviour is based on a number of factors. The most important factor is goal or several goals associated with the object. During problem solving, an agent in order to exhibit behaviour, may have to select from one or several goals depending on the nature of the dynamic world.

In a dynamic multiagent world, the behaviour is also based on appropriate commitment of the agent to all unexpected situations in the world including state changes, failures, and other agents' mental and physical behaviours. An agent intending to achieve a goal must first commit itself to the goal by assigning the necessary resources, and then carry out the commitment when the appropriate opportunity comes. Second, if the agent is committed to executing its action, it needs to know how weak or strong the commitment is. If he commitment is week, the agent may not want to expend too much of its resources in achieving the execution. The agent thus needs to know the degree of its commitment towards the action. This degree of commitment quantifies the agent's attitude towards the action execution. For example, if the agent considers the action execution to be of higher importance (an attitude towards the action), then it may choose to execute the action with greater degree of commitment; otherwise, the agent may drop the action even when it had failed at the first time. Thus, in our formulation, an agent when it performs an activity, since the activity is more likely that it will not succeed in a dynamic world, agents will adopt a definite attitude towards every activity while performing that activity. The adopted attitude will guide the agent in 
responding to failure situations. Also the behaviour must be consistent over the period of time during which the agent is holding the attitude. Thus attitudes, once adopted, must persist for a reasonable period of time so that other agents can use it to predict the behaviour of the agent under consideration. Thus,an agent cannot afford to change its attitude towards a given object too often, because if it does, its behaviour will become somewhat like a reactive agent, and its attitude may not be useful to other agents. Once an agent chooses to adopt an attitude, it strives to maintain this attitude, until it reaches a situation where the agent may choose to drop its current attitude towards the object and adopt a new attitude towards the same object. Attitude is thus defined as follows: An agent's attitude towards an object refers its persistent degree of commitment towards achieving one or several goals associated with the object, which give rise to an overall favourable or unfavourable behaviour with regard to that object.

\subsection{Need for Attitudes in the Multi-agent Dynamic World}

The agents in a static world solve problems by deriving a set of plans and executing these plans. Thus in a static world, the agent will achieve its goal safely as long as it initially chooses the right sequence of actions to execute. In such cases, the agents can engage in blind commitments to the execution of the plan or there is no need to reexamine the commitments. However, in a dynamic world the agent has not only to select an appropriate action to execute, but also must commit to the action before attempting to execute and then execute that action. Executing this mental action becomes more crucial when the action itself is a complex action consisting of several primitive actions. The current approach employed by researchers involves execution monitoring [25] where agents will have to take certain corrective measures whenever a plan is affected by the unexpected changes in the world. This approach is inadequate in a complex multi-agent world because monitoring based on local view about the world and local plans will be difficult. Even permitting agents to communicate with each other for communication purposes will not be adequate unless there is a top level approach to the whole problem of responding to changes in the world first collectively and then individually. Thus when the agent operates in a dynamic environment, blind commitment strategy may not be adequate to achieve a given goal. As argued in [5], an explicit commitment is required to help the agent in filtering out the other problems that may be generated by the world for the agent to solve. However, in the complex multi-agent dynamic world, the notion of commitment is too restrictive, and agents using this notion alone do not necessarily exhibit appropriate complex behaviors.

Many formulations and implementations of agent based system do not address one important issue: that is, when a task is specified to an agent for it to perform, what should the agent do when it finds it impossible to do? This often arises in a dynamic world. In fact, failure is more a rule than an exception in a multi-agent dynamic world. In such a world, typically an agent only has partial knowledge of the world and physical and mental states of other agents. A failure can occur for many reasons under a given mental and physical state of an agent. We will now briefly consider what behavioral options are available to an agent under a failure situation. There are two options available to an agent when it meets with a failure: one to acknowledge the failure and give up the task. Two, to acknowledge the failure, but look for any other ways of achieving the same task, before giving it up. The first option is the traditional way of dealing with failure. However, in a dynamic world, agents cannot afford to give up a task so easily. But now the question arises, in case the agent chooses the second option, how far should the agent go towards achieving the given task? For example, when the agent is executing an action as a part of performing a specified behavior, what should the agent do when the action for some reason fails? Typically, an action may fail because some preconditions do not hold or some invariants that need to be holding during the action execution may not hold at the given time. If the agent has chosen the second option, there may be several ways of reattempting the same action. For example, to attempt the action again, the agent may attempt to achieve the preconditions first, which will affect certain resources. This will also affect the other actions in the plan, or violate certain planner assumptions used by other agents. Now the agent can choose to ignore these effects, which can become costly afterwards. In the case when other actions are affected, it may at that point ignore the current plan and adopt a different plan. But, if the actions of other agents are affected, fixing them may be more expensive. If the agent is committed to executing its action, it needs to know how weak or strong the commitment is. If the commitment is weak, the agent may not take too much trouble in achieving the execution. But, if the agent's commitment is strong, then it may not mind going to any extreme in achieving the execution. Thus, the agent needs to know the degree of its commitment towards the action. This degree of commitment quantifies the agent's attitude towards the action execution. For example, if the agent considers the action execution to be of higher importance (an attitude towards the action), then it may choose to execute the action with greater degree of commitment; otherwise, the agent may drop the action even when it had failed at the first time. Thus, in our formulation, agents will adopt a definite attitude towards an activity while performing that activity, even if the activity may not succeed in a dynamic world. The adopted attitude will guide the agent in responding to failure situations. Thus, ideally, agents in dynamic worlds must adopt suitable attitudes while engaging in an activity.

\subsection{Properties of Attitudes}

We denote the attitude by $\mathbf{K}$, thus $\mathbf{K}_{\mathrm{A}}(\mathrm{x})$ denotes that an agent $\mathrm{A}$ has an attitude $\mathbf{K}$ towards the object $\mathrm{x}$, where $\mathrm{x}$ can be either an agent or an object (physical or mental). The attitudes adopted by agents must exhibit three important properties: explicitness, evaluational consistency and persistence. 


\subsubsection{Explicitness}

In a multi-agent dynamic world, an attitude $\mathbf{K}$ that an agent $\mathrm{A}$ holds towards an object $\mathrm{x}$, that is $\mathrm{K}_{\mathrm{A}}(\mathrm{x})$, must be explicit. We define explicitness as follows: $A n$ attitude $K$ held by an agent $A$ is said to be explicit if the agent $A$ has the capability to inform other agents that it believes that it is (indeed) holding the attitude $K$ towards $x$ whenever it is holding that attitude. Thus, according to this definition it is sufficient that agent $A$ exhibits the above minimum communicative behaviour. If the attitude is implicit, then there is no way of finding out whether any such attitude exists at all, since the agent may not even have a name to refer to that attitude. Since the mental states are entirely local, the attitudes of one agent may not be entirely visible to another agent or implicit. An observer can at most observe the behaviors of the agent, infer a certain attitude, name it, and describe it to the other agents. In a multiagent world, a given agent $A_{1}$ will have to be constantly interacting with other agents, say $A_{2}$. This is the case not only in cooperative situations, but also in other noncoopertive situations as well (for example, competing, indifferent, etc.). In some simple cases, it may be possible for an observing agent $A_{2}$ to infer partial information about the mental states of $A_{1}$ (called mental state recognition).

\subsubsection{Attitudes and Behavior}

The attitude is typically viewed as a latent or underlying variable that is assumed to guide or influence behavior. The tie-up between attitudes and behavior can be noticed if one considers real life situations. For example, an individual agent with an attitude toward the victim in a fire world will help the victim on one occasion and pass without offering help on another occasion. Thus the totality of agent's attitude ultimately determines its overall behavior or the knowledge of an agent's attitude, therefore, permits prediction of one or more specific behaviors. The change in behavior is also linked with attitudes. The behavior of an agent changes if there is a change in attitude towards that behavior.

When an attitude is adopted, the agent has to exhibit an appropriate behaviour. There are several requirements for this behaviour. First, in a dynamic multi-agent world, this behaviour must include an appropriate response of the agent to all unexpected situations in the world including state changes, failures, and other agents' mental and physical behaviours which are inferred through messages received and by recognition. (In a collective activity, there are several kinds of messages possible.) Second, the behaviour must be consistent over the period of time during which the agent is holding the attitude. For example, if the agent is holding the view importantA(t) towards a toy $t$, during a time interval $\mathrm{T}$, then for some brief sub-interval of $\mathrm{T}$ it may become necessary to drop this attitude in favour of some other attitude $\mathbf{K}$, and later substitute $\mathbf{K}$ by important attitude and repeat this behaviour as often as the agent may deem it necessary. However, the fundamental requirement is that over the entire period of time $T$, there must be an overall consistency in the complete behaviour of the agent, which may be judged as appropriate (for the adopted attitude) by the fellow agents in society.

\subsubsection{Attitudes and Persistence}

The attitudes, once adopted by an agent $A$, must persist for a reasonable period of time so that other agents can use it to predict the behavior of the agent $A$. An agent cannot thus afford to change its attitude towards a given object every time it senses a change in the world, because if it does, its behavior will become somewhat like a reactive agent, and recognize that this attitude may not be useful to other agents in complex worlds. Thus, perhaps the most important requirement of an attitude is that it should be persistent for a reasonable period of time. This means, the agents' mental and physical behavior should be such that any other distractions that an agent is likely to face should be postponed holding on to the current attitude. For example, when the agent holds the attitude important $_{A}(\mathrm{t})$, it has to engage itself in a behavior that conveys the meaning of this attitude. This is done by forming a set of intentions resulting in a set of commitments. Note that the set of intentions and commitments should necessarily include the persistence requirements of the attitude as well. Thus, if the attitude important implies $_{A}(\mathrm{impl}$ ) particular plan $\mathrm{p}$ has to be executed, then the agent will execute this plan overcoming the hurdles if any that the agent may face during the execution. To what extent the agent will venture to face the hurdles to achieve the persistence is essentially included in the behavioral requirements of the attitude important $\boldsymbol{i}_{A}(\mathrm{t})$. Thus inherent in the attitude specification are the requirements for the persistence of the attitude as well. Once an agent chooses and adopts an attitude, it strives to maintain this attitude, until it reaches a situation where the agent may choose to drop its current attitude towards the object and adopt a new attitude towards the same object. Complex agents, before changing to new attitudes, may sometimes choose to vary the degree of attitude as deemed fit for the current environmental condition before entirely dropping it and adopting a new one.

\subsection{Computational Model of Attitude}

We present a computational model in which we define that every attitude $\mathbf{K}$ towards an object $x$ exhibits a behavior beh consisting of physical, communicative and mental actions. We represent the attitude $\mathbf{K}(\mathrm{x})$ using the following attributes:

Name of Attitude: This attribute describes the name of the attitude e.g. like, hate, cautious etc. 
Description of Object: The description of the object contains the name of the object and a description of the internal organization in terms of the components of the object.

Basic agent behavior towards $x$ : This attribute specifies the behavior that will be performed by the agent with respect to the object $x$.

Evaluation: This attribute specifies whether the attitude is favorable or not. If the beliefs of an agent are with favorable attributes, the attitude tends to be positive. Conversely, a negative attitude will result if the beliefs have primarily unfavorable attributes.

Concurrent attitudes: This attribute specifies any other attitudes that can coexist with this attitude.

Persistence of Attitude: This attribute specifies how long the attitude will persist under various situations. For example, it may specify how the attitude itself will change over time; that is, when to drop it and change it to another attitude, when to pick it up and how long to maintain it.

Type of Attitude: This attribute specifies whether the attitude is individual or collective.

All the attributes described above play an important part in the proper understanding of the agents' behaviors in a multiagent dynamic world. Without the knowledge of these attributes, the agent will not be able to respond appropriately to the various situations of the dynamic world.

\section{Attitude Based Bidding Strategy}

The bidding process has been long modelled using the tools of game theory [14], [17], and these are now being used extensively in the development of software agents for automated negotiation. In such encounters, each agent has to make decisions about generating offers and counter offers in such a way that its own utility from the final agreement is maximized. An essential input to his decision making process is information; here defined as the knowledge about all factors which affect the ability of an individual to make choices in a given situation. The bidder agent operates effectively in the marketplace, only if it possesses a strategy, which ensures that it can obtain the item within the given time in a manner really consistent with the consumer's preferences. An agent's bidding strategy is the specification of the offers the agent plans to make during the auction and it also has the consumer's attitudes towards the item it wants to procure in an auction.

In this paper, agents' attitudes are the ultimate component of the bidding strategy that decides about placing or accepting offers, making counter offers or withdrawing from an auction. We say that, when agents are participating in an auction, they have appropriate attitudes towards different criteria the agents have to acquire in order to buy an item in an auction. Thus when an agent participates in an auction, an attitude prescribes how to perform precisely till the item is acquired and the auction is finished. The bidder agent registers itself in a particular auction that sells the product which it wants to buy. It then gathers relevant information about the auction i.e. the start time of auction, end time of auction and current bid values in that auction. It then calculates the maximum bid it is willing to make at a particular time in that auction. To determine the current maximum bid, the agent considers several bidding criteria like price of item, time left to negotiate, quality of item etc. Towards each bidding criteria, the agent can have different attitudes. Let $B=\left\{b_{1}, b_{2}, \ldots, b_{n}\right)$ be a given set of finite bids for an item in an auction and $C=\left\{C_{1}, C_{2}, \ldots C_{m}\right)$ be given set of finite criteria; and $D=\left\{D_{1}, D_{2} . . D_{1}\right\}$ be a given set of agent bidders. The steps for selecting the best possible bid are as follows:

Step1: A decision maker (agent) determines the weight of selection criteria $\mathrm{C}$ using the Analytic Hierarchy Process method (AHP) [23]. Each bidding constraint have different weight depending upon its importance at particular time.

The relative importance of a selection criterion is given by pair-wise comparison and a comparison matrix is established. The comparison scale ranges from 1to 9, each representing the concepts of: 1-equally important;2weakly more important; 5-strongly more important;7-demonstratively more important;9-absolutely more important,2,4,6,8 are intermediate values between adjacent adjustments. Consistent weights for every selection criteria can be determined by calculating the geometric mean of each row of matrix and then the result numbers are normalized. The weights are denoted as $w_{1}, w_{2}, \cdots, w_{m}$, where $w_{i} \in[0,1], i=1,2, \cdots, m$ and $\sum_{i=1}^{m} w_{i}=1$.

Step 2: Against each selection criterion, assign "1" to the preferred bid and " 0 " to the unwanted bid. As the choice is a bidder's selective judgment, agents may often find themselves in a situation where it is difficult to choose or reject a bid. Thus attitudes are introduced to express the possibility of selecting a bid under selection criteria.

For each such bidding constraint, there is a corresponding attitude that suggests the value to the bid based on the criterion at that time. An attitude can be introduced to express the possibility of selecting a bid $i$ under criterion $j$ for a decision maker agent $k$. In a decision making process, the attitudes $a_{i j}{ }^{k}(i=1,2, . ., n, j=1,2, \ldots, m, k=1,2, . ., l)$ belongs to a 
set of linguistic terms [31] that contain various degree of preferences required by a decision maker agent $D_{k}(k=1,2, \ldots, l)$. As each bidding criterion play different role, the attitude towards each criterion varies from low to high or vice versa. The reasoning to calculate maximum bid utilizes fuzzy rules, as they can very well cope with uncertainties. We use seven linguistic terms for attitudes. The attitude towards each bidding constraint can be described in linguistic terms, such as "very low", "low", "medium", "high", and "very high", and can be described by triangular fuzzy numbers (Table 1). The attitudes are represented by attitude matrix $\left(a_{i j}\right)^{k}{ }_{n \times m}$.

Table1: Linguistic variables for attitudes

\begin{tabular}{|l|l|}
\hline Linguistic Variables & Fuzzy Numbers \\
\hline Very Low $(\mathrm{VL})$ & $(0,0,0.1)$ \\
\hline Low $(\mathrm{L})$ & $(0,0.1,0.3)$ \\
\hline Medium Low $(\mathrm{ML})$ & $(0.1,0.3,0.5)$ \\
\hline Medium (M) & $(0.3,0.5,0.7)$ \\
\hline Medium high $(\mathrm{MH})$ & $(0.5,0.7,0.9)$ \\
\hline High $(\mathrm{H})$ & $(0.7,0.9,1)$ \\
\hline Very high $(\mathrm{VH})$ & $(0.9,1,1)$ \\
\hline
\end{tabular}

Step 3: The attitude matrix is aggregated to attitude vector $A_{i}(i=1,2, \ldots, n)$ :

$$
A_{i}{ }^{k}=w_{1}{ }^{k} * a_{i 1}{ }^{k}+w_{2}{ }^{k} * a_{i 2}{ }^{k}+\cdots+w_{n}{ }^{k} * a_{i n}{ }^{k} \text {. }
$$

Step 4: Considering all the bidders in an auction equally important, a weighted normalized fuzzy decision vector is created. The normalized weight of bidder $D_{k}(k=1,2, . ., I)$ is donated by $\left(v_{1}, v_{2}, . ., v_{1}\right)$.

$$
\left(r_{1}, r_{2}, . . r_{n}\right)=\left(v_{1}, v_{2}, \ldots, v_{l}\right)\left(\begin{array}{cccc}
a_{1}{ }^{1} & a_{2}{ }^{1} & . . & a_{m}{ }^{1} \\
a_{1}{ }^{2} & a_{2}^{2} & . \cdot & a_{m}^{2} \\
\ldots & . . & . . & . . \\
a_{1}{ }^{n} & a_{2}{ }^{n} & . . & a_{m}^{n}
\end{array}\right)
$$

Step 5: In the weighted normalized fuzzy decision vector the elements are normalized into positive triangular fuzzy numbers, and their ranges belong to the closed interval $[0,1]$. Calculate fuzzy positive and negative solution distances. Define a fuzzy positive-ideal solution (FPIS, $r^{*}$ ) and a fuzzy negative-ideal solution (FNIS, $r$ ) as:

$$
r^{*}=(1,1,1) \text { and } r^{-}=(0,0,0) \text {. }
$$

The distance between each $A_{i}$ and $r^{*}, A_{i}$ and $r^{*}$ can be calculated as:

$$
d_{i}^{*}=d\left(A_{i}, r^{*}\right) \text { and } d_{i}^{-}=d\left(A_{i}, r^{-}\right),
$$

where $d(.,$.$) and i=1,2, \cdots, n$, is the distance measurement between two fuzzy numbers. . Many functions of distance measurement have been proposed, but the vertex method proposed here is an effective and simple method for calculating the distance between two triangular fuzzy numbers [8].

\section{REPEAT}

1. Input the weight of each bidding criteria.

2. Determine the bidder's attitude at that time based on each criteria using the linguistic terms.

3. Aggregate attitudes for possible bids

4. Calculate positive/negative fuzzy distances.

5. Calculate closeness coefficient to find best possible bid.

UNTIL item is acquired or auction is closed

Figure 2: Procedure for Attitude Based Bidding Strategy

Step 6: A close coefficient is defined to determine the ranking order of all bids values once the $d_{j}^{*}$ and $d_{j}{ }^{-}$of each bid $B_{i}(i=1,2, . ., n)$ are obtained. The closeness coefficient of each bid is calculated as

$$
C C_{i}=\frac{1}{2}\left(d_{i}^{*}+\left(1-d_{i}^{-}\right)\right), \quad j=1,2, \cdots, n .
$$


The bid that corresponds to the maximum closeness coefficient is the best bid for the item at that time of the auction. The agent's decision-making bidding strategy is summarized in Figure 2.

\section{A Numerical Example}

Suppose there are three bidders $D=\left(D_{1}, D_{2}, D_{3}\right)$ in an auction. They have three bids $B=\left(B_{1}, B_{2}, B_{3}\right)$ under four selection criteria $C=\left\{C_{1}, C_{2}, C_{3}, C_{4}\right\}$ for choosing a particular item in an auction. To make a most satisfactory bid for the item, the following steps are:

Step1: By using the AHP method, first establish the comparison matrix for the selection criteria:

$$
\begin{aligned}
& E^{1}=\left(\begin{array}{cccc}
1 & 1 & 1 / 2 & 1 / 2 \\
1 & 1 / 2 & 1 & 1 / 3 \\
2 & 1 & 2 & 1 \\
2 & 1 & 3 & 1
\end{array}\right) \Rightarrow\left(\begin{array}{l}
0.1635 \\
0.1477 \\
0.3270 \\
0.3618
\end{array}\right) \Rightarrow\left(\begin{array}{l}
w_{1}^{1} \\
w_{3}^{1} \\
w_{4}^{1}
\end{array}\right) \\
& E^{2}=\left(\begin{array}{cccc}
1 & 1 & 1 & 1 \\
1 & 1 / 2 & 1 & 1 / 2 \\
2 & 1 & 1 / 2 & 1 / 3 \\
2 & 3 & 1 / 2 & 1
\end{array}\right) \Rightarrow\left(\begin{array}{l}
0.2643 \\
0.1869 \\
0.2008 \\
0.3479
\end{array}\right) \Rightarrow\left(\begin{array}{l}
w_{1}^{2} \\
w_{2}^{2} \\
w_{3}^{2} \\
w_{4}^{2}
\end{array}\right) \\
& E^{3}=\left(\begin{array}{cccc}
1 & 1 / 2 & 1 / 3 & 1 \\
1 / 2 & 2 & 1 / 3 & 1 \\
1 / 4 & 1 & 2 & 3 \\
4 & 3 & 2 & 1
\end{array}\right) \Rightarrow\left(\begin{array}{l}
0.1354 \\
0.1610 \\
0.2345 \\
0.4691
\end{array}\right) \Rightarrow\left(\begin{array}{l}
w_{1}^{3} \\
w_{2}^{3} \\
w_{3}^{3} \\
w_{4}^{3}
\end{array}\right)
\end{aligned}
$$

Step 2: Three attitude matrixes are created for 3 possible bids

$$
\begin{aligned}
& \left(\begin{array}{llll}
a_{11}^{1} & a_{12}^{1} & a_{13}^{1} & a_{14}^{1} \\
a_{21}^{1} & a_{22}^{1} & a_{23}^{1} & a_{24}^{1} \\
a_{31}^{1} & a_{32}^{1} & a_{33}^{1} & a_{34}^{1}
\end{array}\right)=\left(\begin{array}{cccc}
L & V L & H & M \\
H & V H & M & M L \\
V H & L & H & V L
\end{array}\right) \\
& \left(\begin{array}{llll}
a_{11}^{2} & a_{12}^{2} & a_{13}^{2} & a_{14}^{2} \\
a_{21}^{2} & a_{22}^{2} & a_{23}^{2} & a_{24}^{2} \\
a_{31}^{2} & a_{32}^{2} & a_{33}^{2} & a_{34}^{2}
\end{array}\right)=\left(\begin{array}{cccc}
M & L & H & M \\
L & V H & M & M L \\
V H & M & H & V L
\end{array}\right) \\
& \left(\begin{array}{llll}
a_{11}^{3} & a_{12}^{3} & a_{13}^{3} & a_{14}^{3} \\
a_{21}^{3} & a_{22}^{3} & a_{23}^{3} & a_{24}^{3} \\
a_{31}^{3} & a_{32}^{3} & a_{33}^{3} & a_{34}^{3}
\end{array}\right)=\left(\begin{array}{cccc}
M & L & V L & M \\
L & V H & M H & M L \\
V H & M & L & V L
\end{array}\right)
\end{aligned}
$$

Step 3: Aggregating belief vectors

$$
\begin{aligned}
& A_{1}{ }^{1}=w_{1}{ }^{1 *} a_{11}{ }^{1}+w_{2}{ }^{1 *} a_{12}{ }^{1}+w_{3}{ }^{1 *} a_{13}{ }^{1}+w_{4}{ }^{1 *} a_{14}{ }^{1} \\
& =0.1635^{\star}(0,0.1,0.3)+0.1477^{*}(0,0,0.1)+0.3270^{\star}(0.7,0.9,1)+0.3618(0.3,0.5,0.7) \\
& =(0.3374,0.4916,0.6441) \\
& A_{2}{ }^{1}=w_{1}{ }^{1 *} a_{21}{ }^{1}+w_{2}{ }^{1} a_{22}{ }^{1}+w_{3}{ }^{1 *} a_{23}{ }^{1}+w_{4}{ }^{1 *} a_{24}{ }^{1} \\
& =0.1635^{*}(07,0.9,1)+0.1477^{*}(0.9,1,1)+0.3270^{*}(0.3,0.5,0.7)+0.3618(0.1,0.3,0.5) \\
& =(0.3817,0.5668,0.7205) \\
& A_{3}{ }^{1}=w_{1}{ }^{1} * a_{31}{ }^{1}+w_{2}{ }^{1} * a_{32}{ }^{1}+w_{3}{ }^{1 *} a_{33}{ }^{1}+w_{4}{ }^{1 *} a_{34}{ }^{1} \\
& =0.1635^{\star}(0.9,1,1)+0.1477^{\star}(0,0.1,0.3)+0.3270 *(0.7,0.9,1)+0.3618(0,0,0.1) \\
& =(0.3760,0.4726,0.5710)
\end{aligned}
$$




$$
\begin{aligned}
& A_{1}{ }^{2}=w_{1}{ }^{2 *} a_{11}{ }^{2}+w_{2}{ }^{2 \star} a_{12}{ }^{2}+w_{3}{ }^{2 *} a_{13}{ }^{2}+w_{4}{ }^{2 \star} a_{14}{ }^{2} \\
& =(0.2449,0.3811,0.7104) \\
& A_{2}{ }^{2}=w_{1}{ }^{2 \star} a_{21}{ }^{2}+w_{2}{ }^{2 \star} a_{22}{ }^{2}+w_{3}{ }^{2 \star} a_{23}{ }^{2}+w_{4}{ }^{2 \star} a_{24}{ }^{2} \\
& =(4482,0.6296,0.7658) \\
& A_{3}{ }^{2}=w_{1}{ }^{2 *} a_{31}{ }^{2}+w_{2}{ }^{2 *} a_{32}{ }^{2}+w_{3}{ }^{2 \star} a_{33}{ }^{2}+w_{4}{ }^{2 *} a_{34}{ }^{2} \\
& =(0.3785,0.4637,0.5560) \\
& A_{1}{ }^{3}=w_{1}{ }^{3 *} a_{11}{ }^{3}+w_{2}{ }^{3 *} a_{12}{ }^{3}+w_{3}{ }^{3 *} a_{13}{ }^{3}+w_{4}{ }^{3 *} a_{14}{ }^{3} \\
& =(0.3048,0.4591,0.6196) \\
& A_{2}{ }^{3}=w_{1}{ }^{3 *} a_{21}{ }^{3}+w_{2}{ }^{3 *} a_{22}{ }^{3}+w_{3}{ }^{3 *} a_{23}{ }^{3}+w_{4}{ }^{3 *} a_{24}{ }^{3} \\
& =(0.3620,0.5406,0.6952) \\
& A_{3}{ }^{3}=w_{1}{ }^{3 *} a_{31}{ }^{3}+w_{2}{ }^{3 *} a_{32}{ }^{3}+w_{3}{ }^{3 *} a_{33}{ }^{3}+w_{4}{ }^{3 *} a_{34}{ }^{3} \\
& =(0.2866,0.3626,0.4651)
\end{aligned}
$$

Step 4: The three agents have equal weights: $v_{1}=v_{2}=v_{3}=0.333$. The fuzzy decision vectors are

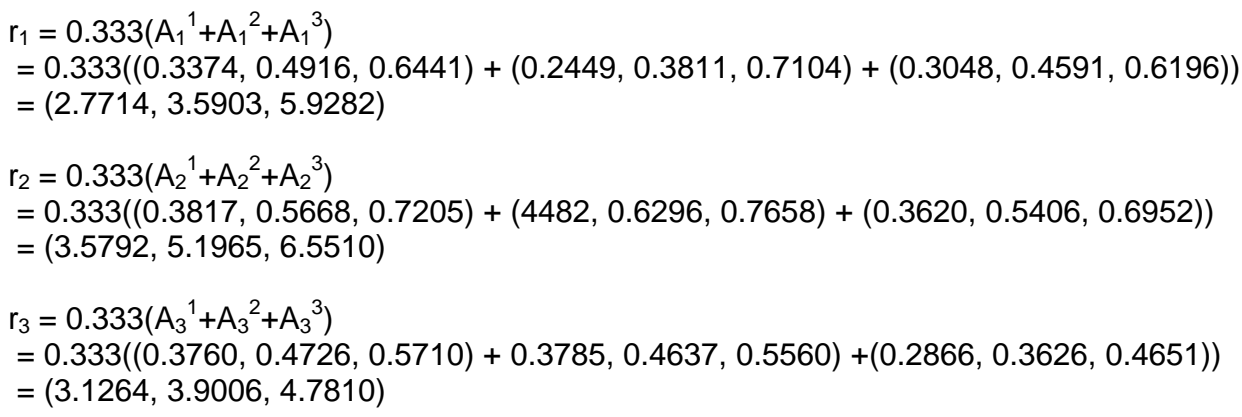

Step 5: We get fuzzy positive are negative solution distances:

$$
\begin{aligned}
d_{1}^{*} & =d\left(r_{1}, r^{*}\right) \\
& =\sqrt{\frac{1}{3}\left[(2.7714-1)^{2}+(3.5903-1)^{2}+(5.9282-1)^{2}\right]} \\
& =3.0640 \\
d_{2}^{*} & =d\left(r_{2}, r^{*}\right)=4.2670 \\
d_{3}^{*} & =d\left(r_{3}, r^{*}\right)=3.0123 \\
d_{1}^{-} & =d\left(r_{1}, r-\right) \\
& =\sqrt{\frac{1}{3}\left[2.7714^{2}+3.5903^{2}+5.9282^{2}\right]} \\
& =4.2980 \\
d_{2}^{-} & =d\left(A_{2}, r-\right)=5.2538 \\
d_{3}^{-} & =d\left(A_{3}, r-\right)=3.9936 .
\end{aligned}
$$


Step 6: Finally, we have

$$
\begin{aligned}
C C_{1} & =\frac{1}{2}\left(d_{1}^{-}+\left(1-d_{1}^{*}\right)\right) \\
& =\frac{1}{2}(4.2980+(1-3.0640))=1.117 \\
C C_{2} & =\frac{1}{2}\left(d_{2}^{-}+\left(1-d_{2}^{*}\right)\right) \\
& =\frac{1}{2}(5.2538+(1-4.2670))=0.9934 \\
C C_{3} & =\frac{1}{2}\left(d_{3}^{-}+\left(1-d_{3}^{*}\right)\right) \\
& =\frac{1}{2}(3.0036+(1-3.0123))=0.9906
\end{aligned}
$$

Since $C C_{1}$ is higher than $C C_{2}$ and $C C_{3}$, a most satisfactory bid for the item is the first bid.

\section{Experimental Evaluation}

We have developed a Trading agent called Atti-TA, which uses our developed bidding strategy at its core. Atti-TA is an adaptive agent that varies its bidding strategy according to the attitude of the agent in the prevailing market conditions. To evaluate the performance of bidding an agent, we undertook an empirical evaluation to show that our bidding strategy performs effectively in a wide range of bidding contexts. To do this, we devised two competitor bidding agents that adopt strategies consistent with classes of behaviour that was observed in the electronic market. In order to assess the robustness of our bidding agents, the experiments have concentrated on evaluating the performance of attitude-based agents as well as agents with no attitude in case of ever changing market conditions. When a problem occurs, the attitude model stipulates a new set of behaviors for the agents. The agents with attitude respond to changes in the world by adopting a set of attitudes towards these changes. The agents without attitude are very slow to respond to the changing market conditions. We have tried to find the probability of success in case (i) agents (A1 and A2) having attitude (ii) agents ( 1 1 and $A 2$ ) having no attitude. The performance of two types of agents is measured in terms of the rate of change of world and time taken to complete the task. Figure 3 shows the performance of agents in terms of their success rate. The success rate is defined as the number of times, as a percentage, the agent is successful in obtaining the item. The $x$-axis of the graph shows success rate in percentage, while the $y$-axis represents the time taken by bidder to acquire an item.

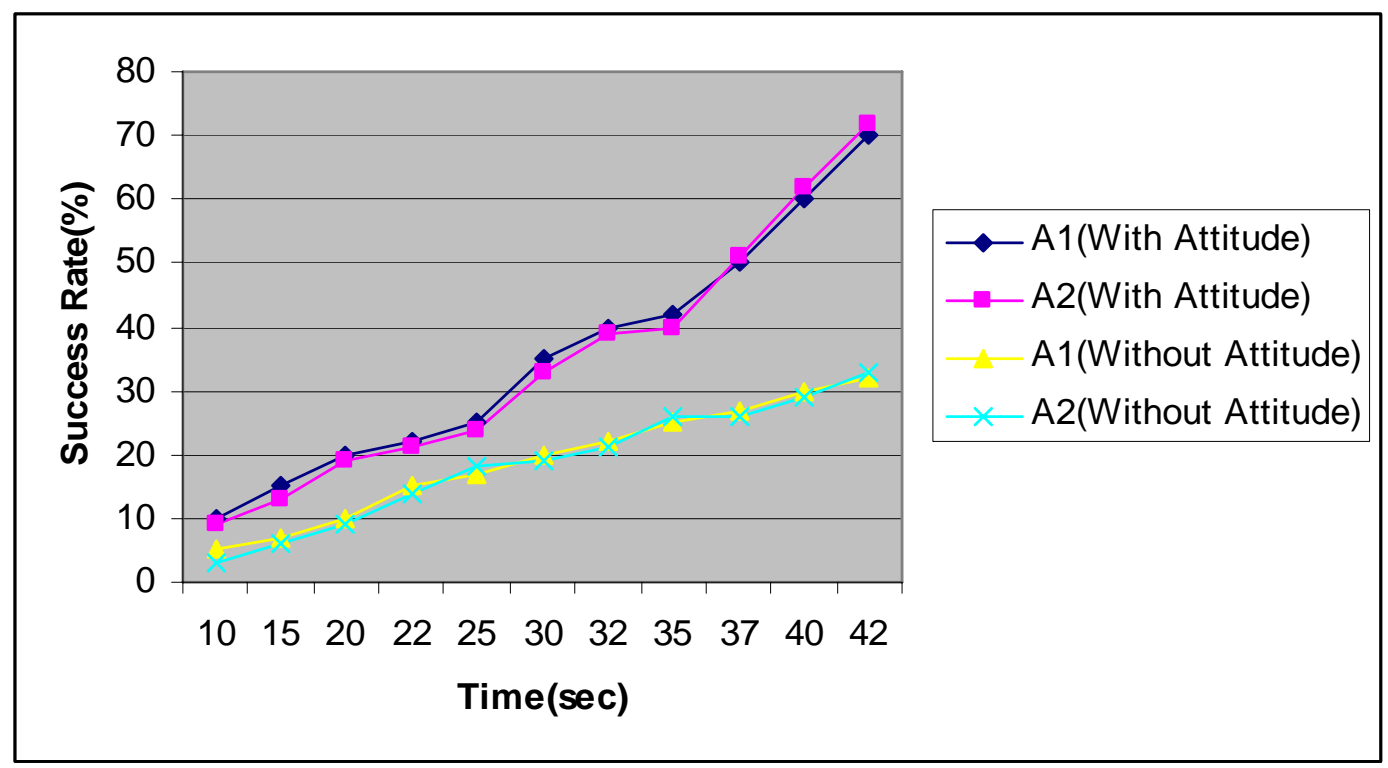

Figure 3: Success Rate Comparisons for agents with or without Attitude 
We also found that success rate in case of attitude-based agents are more, while in case of non-attitude based agents is less. The attitude-based agents performs better than the non-attitude based agents, because attitude based agents can easily adapt themselves to newer situations and have better tools to calculate bid prices appropriately.

We have also tried to find the the success rate for the three agent behaviours i.e. desperate, bargain and balance (Figure 4). It can be seen that agent's with attitude achieve a high success rate as compared to the agents without attitude. This shows that bidders with attitude have strategies to choose from as compared to agents without attitude. The agent's behavior can be categorized as desperate, looking for bargain and balance of both. At any given time, the agent may consider any of these bidding constraints individually or combine them depending on the situation. For example, if the agent considers desire for a bargain as the only bidding constraint, it should start bidding at low value and slowly move towards its private valuation. On the other hand, balanced agent would start bidding at a reasonably high value and slowly reach its private value. The desperate agent would start bidding at a very high value and reach its private valuation very quickly. Thus the success rate of agent having a desperate behaviour is higher than the agent looking for a bargain.

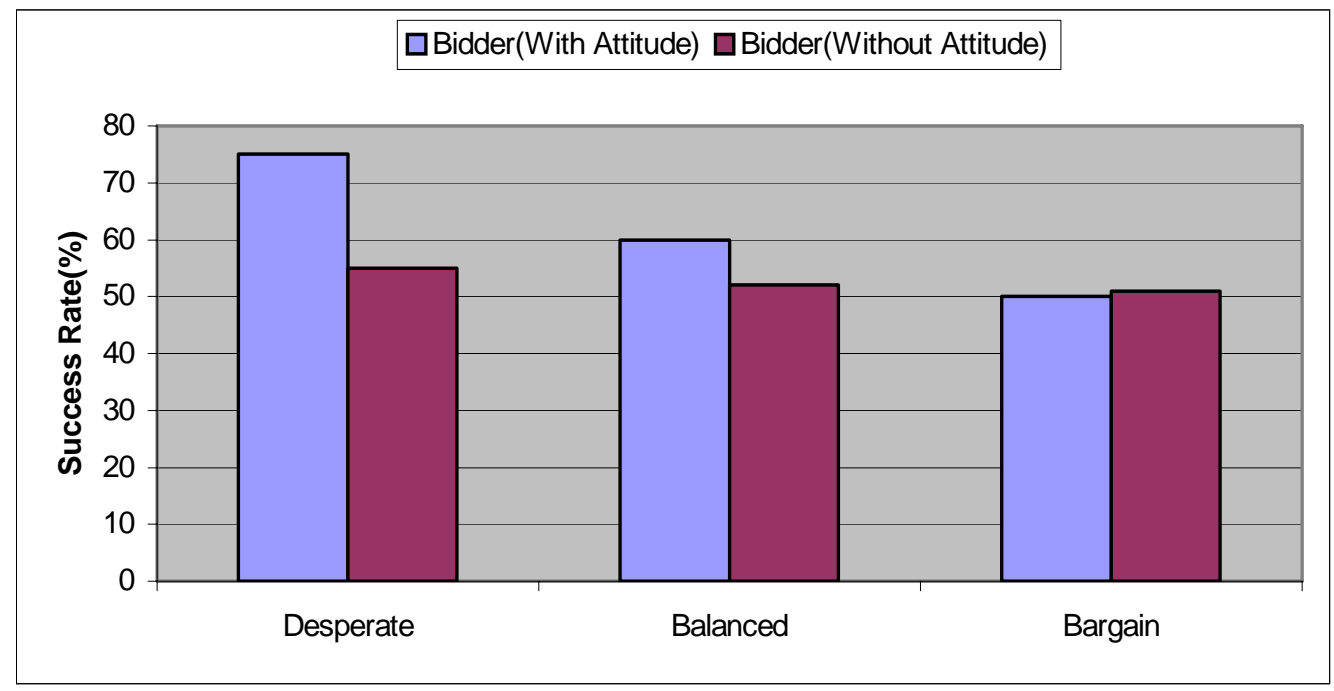

Figure 4: Success rates of Desperate, Balanced and Bargain agents

\section{Conclusions}

In this paper, a novel application of attitude-based agent's bidding strategy in electronic auctions is presented. It details the design, implementation and evaluation of successful attitude based trading agent that employs a range of fuzzy techniques at its core. It was noticed that attitude based agents outperform the agents without attitude in highly dynamic bidding environment. Agents, which adopt attitudes, behave more flexibly and efficiently than agents without attitude and adapt more easily to dynamic situations. Another unique idea presented in this paper is that to deal quantitatively the imprecision or uncertainty of attitudes, fuzzy theory is used. Our fuzzy reasoning methods for predicting closing prices can be reused in multiple auction applications in which an agent tries to procure a good from a number of inter-related auctions that are running concurrently. The fuzzy reasoning methods provide attitude based agents provide resources in the decision making process of bidding agent. The bidding strategy also allows for flexible heuristics both for the overall gain and for individual attribute evaluations. Due to the uncertainty in electronic auctions, it is difficult to use probability based method to calculate the correlation between auctions because assigning prior probabilities is often impossible in practice. However, our fuzzy reasoning is intuitive and is readily comprehensible to the agent's designers. For example, when faced with multiple inter-related auctions, the following factors can be used in the prediction rules: the auction closing time, the goods purchased, the current ask price for a given auction, the current winning bid and so on. The proposed fuzzy decision making framework integrates three properties: bidders may have different weights; bidders can express fuzzy preferences for alternatives solutions, the bidders can give different judgments on solution criteria. Each of these aspects has been studied on an individual basis resulting in extensive literature on the subject. However, there is no research done on combining the three properties in a method designed to deal with linguistic terms describing individual bidder's preferences using fuzzy triangle numbers.

Our attitude based agents has been successful across a wide range of environments (our controlled experiments). Naturally the strategies that have been employed are tailored to the specific auction context. Nevertheless, we believe that our domain exhibits a number of characteristics that are common to many real-world, online trading environments. These attributes include a time constrained environment, network latency, unpredictable opponents, multiple auction types, and the need to purchase inter-related goods. Given this, we 
believe that a number of technologies and insights from our work are applicable in a broader agent-mediated ecommerce context and our future work aims to exploit these.

There are a number of areas of further investigation. We would also like to compare our bidding techniques with other decision theoretic approaches to determine the relative strengths and weaknesses of these methods. Different strategies may perform well in some environments but may perform poorly in another. The numbers of strategies that can be employed are endless and the search space is huge. To address this issue, we intend to use learning techniques to obtain a model of the price dynamics based on the past data and to search for most successful strategies in predefined environments in an offline fashion. Furthermore the pattern recognition or data mining can be used to classify the environment i.e. electronic auction by integrating the decision process with further possible representative of the trading evolution.

\section{References}

[1] P. Anthony and N. R. Jennings, Evolving bidding strategies for multiple auctions, in Proceedings of 15th European Conference on Artificial Intelligence, Netherlands, 2002, pp. 187-182.

[2] P. Anthony and N. R. Jennings, Developing a bidding agent for multiple heterogeneous auctions, ACM transactions on Internet Technology, vol. 3, no. 3, pp. 185-217, 2003.

[3] M. E. Bratman. Intentions, Plans and Practical Reason. Harvard University Press, Cambridge, MA, 1987.

[4] M. Bitchler. The Future of E-Markets: Multi-Dimensional Market Mechanisms. Cambridge University Press, 2001.

[5] M. E. Bratman, D. J. Israel, and M.E.Pollack. Plans and Resource-bounded Practical Reasoning. Computational Intelligence, 4. pp. 349-355, 1988.

[6] A. Byde, C. Priest, and N. R. Jennings. Decision procedures for multiple auctions. In Proceedings of the First International Joint Conference on Autonomous agents and Multiagent Systems, Bologana, Italy, July 2002, pp. 613-620.

[7] P. R. Cohen and H. J. Levesque. Teamwork. Special Issue on Cognitive Science and Artificial Intelligence, vol. 25, no. 4, 1991.

[8] C. T. Chen. Extensions of the TOPSIS for Group Decision making Under Fuzzy Environment. Fuzzy Sets and Systems, vol 1, no. 114, pp. 1-9, 2000.

[9] M. Fishbein and I. Ajzen. Belief, Attitude, Intention and Behaviour: An Introduction to theory and research. Reading, MA, USA: Addison-Wesley, 1975.

[10] T. Fraichard and P. Garnier, Fuzzy control to drive car like vehicles, Robot. Auto. System. vol.34, no.1, pp.1-22, 2001.

[11] P. Faratin, C. Sierra and N. R. Jennings. Negotiation decision functions for autonomous agents. International Journal of Robotics and Autonomous Systems, vol. 24, no. 3-4, pp. 159-185,1998.

[12] A. Greenwald and P. Stone. Autonomous Bidding Agents in the Trading Agent Competition. IEEE Internet Computing, March 2001, pp. 52-60.

[13] M. He, N. R. Jennings and A. Prügel-Bennett (2006). A heuristic bidding strategy for buying multiple goods in multiple English auctions. ACM Trans on Internet Technology, vol. 6, no. 4 pp. 465-496, 2006.

[14] N. R. Jennings, P. Faratin, A. R. Lomuscio, S. Parsons, C. Sierra and M. Wooldrige. Automated negotiation: prospects, methods and challenges. Group Decision and Negotiation, vol. 10, no. 2, pp. 199-215, 2001.

[15] R. Kowalcyzk, On negotiation as a distributed fuzzy constraint satisfaction problem, in Proceedings DEXA eNegotiation Workshop, 2000, pp. 631-637.

[16] R. Kowalcyzk and V. Bui, On Fuzzy e-negotiation agents: Autonomous negotiation with incomplete and imprecise information, in Proceedings Dexa e-Negotiation Workshop, 2000.

[17] Sarit Kraus. Strategic Negotiation in Multi-agent Environments. MIT Press, 2001.

[18] X. Luo, N. R. Jennings, N. Shadbolt, H. F. Leung and J. H. M. Lee, A fuzzy constraint based model for bilateral, multi-issue negotiation in semi-competitive environments, Artificial Intelligence, vol. 148, no. 1-2, pp. 53-102, 2003.

[19] X. Luo, C. Zhang and N. R. Jennings, A hybrid model of sharing between fuzzy, uncertain and default reasoning models in multi-agent systems, International Journal of Uncertainty, Fuzziness Knowledge Based Systems, vol. 10, no. 4, pp. 401-450, 2002

[20] N. Matos and C. Sierra, Evolutionary computing and negotiating agents, in Agent Mediated Electronic Commerce. New York: Springer-Verlag, 1998, vol. 1571, Lecture Notes in Artificial Intelligence, pp. 126-150.

[21] S. Mohamoaddi, I. Hassanzadeh, R. Mathur and K. Patil, "A new fuzzy decision making procedure applied to emergency electric power distribution scheduling", Engineering Application in Artificial Intelligence, vol.13, no. 6, pp. 731-740, 2000.

[22] C. Priest, C. Bartolini, and I.Philips. Algorithm design for agents which participate in multiple simultaneous auctions. In Agent Mediated Electronic Commerce III, LNAI, pp. 139-154, Berlin, German, 2001.

[23] T. L. Saaty , The Analytic Hierarchy Process, NY, McGraw Hill,1980.

[24] P. Stone, M. L. Littman, S. Singh and M. Kearns, Attac-2000: An adaptive autonomous bidding agent, Journal of Artificial Intelligence Research, vol. 15, pp. 189-206, 2001.

[25] M. Tambe and P. S. Rosenbloom. Architectures for agents that track other agents in multi-agent worlds Intelligent Agents, Vol II Springer Verlag Lecture Notes in Artificial Intelligence (LNAI 1037), 1996.

[26] K. K. Tan and K. Z. Tang, "Vehicle dispatch system based on taguchi-tuned fuzzy rules", European Journal of Operation Research, vol. 128, no. 3, pp. 545-557, 2001. 
[27] Terri C. Walker and Richard K. Miller. 1990. Expert Systems Handbook : An Assessment of Technology and Applications. Englewood Cliffs, NJ: Prentice-Hall.

[28] M. P. Wellman, P. R. Wurman, K. O'Malley, R. Bangera, S. Lin, D. Reeves, and W. E. Walsh. A trading agent competition. IEEE Internet Computing, vol. 5, no. 2, pp. 43-51, April, 2001.

[29] J. F. F. Yao and J. S. Yao, "Fuzzy decision making for medical diagnosis based on fuzzy number and compositional rule of inference". Fuzzy Sets System, vol. 120, no. 2, pp. 351-366, 2001.

[30] D. Zeng and K. Sycara, Bayesian Learning in Negotiation, International Journal Human Computer Studies, vol. 48, pp. 125-141, 1998.

[31] G. Zhang and J. Liu. An integrated group decision-making method dealing with fuzzy preferences for alternatives and individual judgments for selection criteria. Group Discussion and Negotiation, vol. 12, pp. 501515. 